\title{
Association of CYP2D6 polymorphisms and extrapyramidal symptoms in schizophrenia patients receiving risperidone: $a$ retrospective study
}

Takahiro Ito', Kazuhiro Yamamoto', Fuminori Ohsawa', Ikuo Otsuka², Akitoyo Hishimoto², Ichiro Sora², Midori Hirai ${ }^{1}$ and Ikuko Yano ${ }^{1 *}$ (D)

\begin{abstract}
Background: Risperidone is mainly metabolized by cytochrome P450 (CYP) 2 D6 in the liver. The gene encoding CYP2D6 is highly polymorphic. The average steady-state plasma concentration of risperidone active moiety is higher in the CYP2D6 intermediate metabolizers (IMs) compared with that in the extensive metabolizers (EMs). An association between drug-induced extrapyramidal symptoms scale (DIEPSS) score and CYP2D6 polymorphisms has not been reported to date. This study investigates the association of CYP2D6 polymorphisms with the severity of extrapyramidal symptoms in schizophrenia patients receiving risperidone therapy.

Methods: Schizophrenia patients undergoing risperidone treatment were recruited for the study in the Kobe University Hospital. We evaluated extrapyramidal symptoms of schizophrenia using the DIEPSS. CYP2D6*10 and CYP2D6* 14 were analyzed using TaqMan ${ }^{\oplus}$ assays, and CYP2D $6 * 5$ was analyzed using the long-PCR method. Patients with CYP2D6*1**5, ${ }^{*} 1{ }^{*} 14,{ }^{*} 5{ }^{*} 10,{ }^{*} 10 /{ }^{*} 10$, and ${ }^{*} 10 /{ }^{*} 14$ were classified as IMs, and patients with CYP2D $6{ }^{*} 1 /{ }^{*} 1$ and ${ }^{*} 1 /{ }^{*} 10$ were classified as EMs. Patients with CYP2D6*5/*5, ${ }^{*} 5{ }^{*} 14$, and ${ }^{*} 14 /{ }^{*} 14$ were classified as poor metabolizers (PMs).

Results: A total of 22 patients were included in the study. No patients were classified as PMs. The dose of risperidone (mg/day) was not significantly different between EMs $(n=15)$ and IMs $(n=7)$ (median with the interquartile range: 4.0 $(2.0-6.0)$ vs. $4.0(2.0-7.0) \mathrm{mg}, p=0.31)$. The age and disease duration of schizophrenia were not significantly different between the EMs and IMs. The DIEPSS score in the IMs was significantly higher than that in the EMs (median with the interquartile range: 5.0 (3.5-6.5) vs. $0.0(0.0-3.0), p<0.001)$. The multiple regression analysis showed that CYP2D6 IMs is a significant risk factor for the DIEPSS $(p<0.05)$.

Conclusion: Special attentions should be paid to the onset of extrapyramidal symptoms in schizophrenia patients identified as CYP2D6 IM undergoing risperidone therapy.
\end{abstract}

Keywords: Schizophrenia, Extrapyramidal symptoms, DIEPSS, CYP2D6, Risperidone

\footnotetext{
* Correspondence: iyano@med.kobe-u.ac.jp

'Department of Pharmacy, Kobe University Hospital, 7-5-2 Kusunoki-cho,

Chuo-ku, Kobe 650-0017, Japan

Full list of author information is available at the end of the article
}

(c) The Author(s). Open Access This article is distributed under the terms of the Creative Commons Attribution 4.0 International License (http://creativecommons.org/licenses/by/4.0/), which permits unrestricted use, distribution, and reproduction in any medium, provided you give appropriate credit to the original author(s) and the source, provide a link to the Creative Commons license, and indicate if changes were made. The Creative Commons Public Domain Dedication waiver (http://creativecommons.org/publicdomain/zero/1.0/) applies to the data made available in this article, unless otherwise stated. 


\section{Background}

The advent of typical antipsychotic medications such as chlorpromazine revolutionized schizophrenia treatment in the 1950s [1]. Atypical antipsychotics developed after 1960s showed a relatively low frequency of extrapyramidal symptoms. The atypical antipsychotic risperidone has a high binding affinity for both dopamine $\mathrm{D}_{2}$ and serotonin $5-\mathrm{HT}_{2}$ receptors and has a proven efficacy in the treatment of schizophrenic positive and negative symptomatology [2]. In the late years, although the atypical antipsychotics such as clozapine and risperidone that relatively had low frequency of extrapyramidal symptoms are used, many patients develop serious acute adverse effects, such as akathisia, dystonia, and parkinsonism, leading to an impaired quality of life of these patients [3-5]. In addition, insufficient management of the adverse effects due to the antipsychotics increases the patients' mortality [6].

Risperidone is mainly metabolized to the active metabolite 9-hydroxy-risperidone by cytochrome P450 (CYP) 2D6 in the liver [7]. Previous pharmacokinetic studies of risperidone have revealed a large interindividual variability between oral dose and actual plasma concentrations [8]. Physiological factors such as age and body weight have been implicated in this variability. In addition, since the gene encoding CYP2D6 is highly polymorphic [9], $C Y P 2 D 6$ status might affect as well risperidone pharmacokinetics. Four phenotypes of CYP2D6 have been defined as follows: (1) poor metabolizers (PMs), without enzyme activity; (2) intermediate metabolizers (IMs), with reduced enzyme activity; (3) extensive metabolizers (EMs), with normal activity; and (4) ultra-rapid metabolizers, with increased enzyme activity [9]. In the Japanese population, EMs are observed at the highest frequency and include CYP2D6*1/*1 (17.8\%), *1/*2 (11.2\%), " $1 /{ }^{*} 10$ (29.7\%), and $* 2 /{ }^{*} 10(7.7 \%)$ [10]. Genotypes classified into PM is rare in the Japanese population, whereas CYP2D6*1/*5 (6.3\%), *5/ *10 (5.2\%), and ${ }^{*} 10 /{ }^{*} 10$ (14.3\%) classified into IM represent about one quarter [10]. CYP2D6*14 as well as $C Y P 2 D 6 * 5$ are reported as the major defective alleles found in Japanese subjects [11]. The frequencies of CYP2D6*5 and *14 were 6.2 and $2.2 \%$, respectively [11]. The area under the concentration-time curve of risperidone in IMs is reported higher than that in EMs [12]. Findings regarding the association between CYP2D6 polymorphisms and response to risperidone have been conflicting [13]. On the other hand, higher plasma concentrations of risperidone plus 9-hydroxy-risperidone, the active moiety of risperidone, are associated with higher incidence of adverse effects [14]. Thus, CYP2D6 genotyping might be useful in personalizing risperidone therapy in patients with schizophrenia to reduce the incidence of adverse extrapyramidal symptoms.

A drug-induced extrapyramidal symptoms scale (DIEPSS) was developed in Japan in 1994 for evaluating the symptoms seen in psychiatry patients taking antipsychotics [15]. DIEPSS is suitable to evaluate the low incidence of extrapyramidal symptoms occurring during the treatment with atypical antipsychotics such as risperidone $[16,17]$. DIEPSS was reported having high inter-rater and test-retest reliability, and a concurrent validity with other rating scales for extrapyramidal symptoms [18].

In the present study, our objective was to investigate the association of CYP2D6 polymorphisms with the severity of extrapyramidal symptoms in schizophrenia patients receiving risperidone therapy.

\section{Methods \\ Patients}

Patients with schizophrenia defined according to the Diagnostic and Statistical Manual of Mental Disorders (DSM)-IV criteria [19] who received risperidone treatment were recruited for the study between February 2011 and July 2013 from the Kobe University Hospital. Patients were eligible for the study if DIEPSS data were available. Patient information including age, disease duration of schizophrenia, gender, body weight, laboratory data, and history of prescription was collected from the electronic medical records.

\section{Ethics approval}

This study was designed and implemented in accordance with the Declaration of Helsinki and its amendments. The present study was approved by the Ethics Committee of the Kobe University Graduate School of Medicine for Genetic Analysis (No. 57). Written informed consent was obtained from each patient registered in the study.

\section{Evaluation of extrapyramidal symptoms}

The extrapyramidal symptoms in each patient were evaluated using the DIEPSS by the attending psychiatrists in Kobe University Hospital when the patient received risperidone maintenance dose. Since risperidone reaches a steady-state within 2 weeks, we evaluated DIEPSS score after day 14 from the start of risperidone therapy. DIEPSS consists of one global item (overall severity) and eight individual items (gait, bradykinesia, sialorrhea, muscle rigidity, tremor, akathisia, dystonia, and dyskinesia); each item is rated on a five-point scale ( 0 , normal; 1 , minimal; 2 , mild; 3 , moderate; 4 , severe) [15]. The primary endpoint in this study was the sum score of the nine items.

\section{CYP2D6 genotyping}

For each participant, we collected peripheral blood samples in EDTA tubes, which were kept at $-80{ }^{\circ} \mathrm{C}$ until use. Genomic DNA was extracted with the QIAamp DNA Blood Midi Kit ${ }^{\oplus}$ (Qiagen Inc., Valencia, CA, USA) according to the manufacturer's instructions. CYP2D6*10 
(rs1065852) and CYP2D6*14 (rs5030865) were analyzed using commercially available TaqMan ${ }^{\circ}$ assays (Applied Biosystems, Foster, CA, USA). CYP2D6"5 (CYP2D6 deletion) was analyzed using the long-PCR method [20]. The distinction of CYP2D6"5/*10 and "10/*10 was not analyzed in this study. On the basis of the previous study $[9,11]$, patients with $C Y P 2 D 6 * 1 / * 5, " 1 / * 14, * 5 / * 10, * 10 / * 10$, and *10/ *14 were classified as IMs. Patients with $C Y P 2 D 6 * 1 / * 1$ and *1/*10 were classified as EMs. Patients with CYP2D6*5/*5, "5/*14, and "14/"14 were classified as PMs.

\section{Statistical analysis}

All statistical analyzes were performed using SPSS $^{\circ}$ Statistics 24.0 (IBM Japan, Tokyo, Japan). Data are expressed as the number of patients or the median with interquartile range. Fisher's exact tests were used to test the distribution of categorical data, whereas MannWhitney $U$ tests were employed to compare the medians of continuous values between groups. Regression analysis was performed to evaluate the relationship between risperidone dose and DIEPSS score. Multiple regression analysis was performed to identify factors affecting the DIEPSS score, in which the DIEPSS score was defined as the dependent variable, and all the independent variables $(p<0.2)$ defined in the univariate analysis were selected and tested. $P$ values less than 0.05 were considered statistically significant.

\section{Results}

A total of 33 patients were recruited from February 2011 to July 2013 and provided written informed consent. Twenty-two patients whose DIEPSS data were available were eligible for the study. Table 1 shows the demographic and clinical characteristics of 22 schizophrenia patients treated with risperidone. The CYP2D6*1/*1, "1/ $* 5$, $" 1 / * 10$, and $* 5 / 10$ or $* 10 / * 10$ genotypes were detected in 2, 2, 13, and 5 patients respectively. Seven patients with CYP2D6*1/*5, *5/*10, and *10/*10 were classified as IMs, and 15 patients with $C Y P 2 D 6^{*} 1{ }^{*} 1$ and *1/*10 were classified as EMs. CYP2D6"5/*5 genotype and "14 allele were not detected in any patient in this study. The dose of risperidone (mg/day) was not

Table 1 Patient demographics

\begin{tabular}{|c|c|c|c|}
\hline & $\mathrm{EM}(n=15)$ & $\mathrm{IM}(n=7)$ & $p$ \\
\hline Age (years) & $33.0(28.0-43.0)$ & $39.0(29.0-64.0)$ & $0.56^{\mathrm{a}}$ \\
\hline Disease duration of schizophrenia (years) & $12.0(6.0-16.5)$ & $9.0(9.0-40.0)$ & $0.43^{\mathrm{a}}$ \\
\hline Gender: female/male & $7 / 8$ & $4 / 3$ & $0.50^{\mathrm{b}}$ \\
\hline Body weight (kg) & $63.0(55.3-78.0)$ & $64.2(59.2-76.7)$ & $0.96^{\mathrm{a}}$ \\
\hline \multicolumn{4}{|l|}{ Laboratory data } \\
\hline AST (IU/L) & $20(16-23)$ & $24(19-32)$ & $0.31^{\mathrm{a}}$ \\
\hline ALT (IU/L) & $24(14-33)$ & $22(17-94)$ & $0.71^{\mathrm{a}}$ \\
\hline Albumin (g/dL) & $4.5(4.3-4.7)$ & $4.5(4.2-4.5)$ & $0.49^{a}$ \\
\hline Bilirubin (mg/dL) & $0.60(0.50-0.90)$ & $0.60(0.40-0.70)$ & $0.32^{a}$ \\
\hline Serum creatinine (mg/dL) & $0.79(0.69-0.90)$ & $0.74(0.66-0.84)$ & $0.37^{\mathrm{a}}$ \\
\hline eGFR $\left(\mathrm{mL} / \mathrm{min} / 1.73 \mathrm{~m}^{2}\right)$ & $79.3(70.7-91.2)$ & $85.0(66.7-102.6)$ & $0.64^{\mathrm{a}}$ \\
\hline \multicolumn{4}{|l|}{ Antipsychotics } \\
\hline Risperidone dose (mg/day) & $4.0(2.0-6.0)$ & $4.0(2.0-7.0)$ & $0.31^{a}$ \\
\hline Risperidone dose (mg/kg) & $0.065(0.034-0.097)$ & $0.074(0.053-0.112)$ & $0.55^{\mathrm{a}}$ \\
\hline Olanzapine (+/-) & $4 / 11$ & $2 / 5$ & $0.65^{b}$ \\
\hline Levomepromazine (+/-) & $5 / 10$ & $1 / 6$ & $0.35^{b}$ \\
\hline Chlorpromazine equivalent dose (mg/day) & $500(400-1010)$ & $475(250-1800)$ & $0.79^{\mathrm{a}}$ \\
\hline \multicolumn{4}{|l|}{ Other treatments } \\
\hline Number of concomitant drugs & $4(2-5)$ & $7(2-8)$ & $0.31^{a}$ \\
\hline Anticholinergics (+/-) & $7 / 8$ & $5 / 2$ & $0.27^{b}$ \\
\hline Mood stabilizers (+/-) & $4 / 11$ & $3 / 4$ & $0.39^{b}$ \\
\hline Benzodiazepines (+/-) & $12 / 3$ & $6 / 1$ & $0.62^{b}$ \\
\hline
\end{tabular}

Data are expressed as the number of patients or median with the interquartile range in parentheses

Two cases (one case in the EM group, the other case in the IM group) were excluded in the body weight and risperidone dose (mg/kg) data, because information on the body weight was not obtained

${ }^{a}$ Mann-Whitney $U$ test

${ }^{b}$ Fisher's exact test

EM extensive metabolizer, IM intermediate metabolizer, AST aspartate aminotransferase, $A L T$ alanine aminotransferase, eGFR estimated glomerular filtration rate 
significantly different between EM and IM groups (median: $4.0(2.0-6.0)$ vs. $4.0(2.0-7.0) \mathrm{mg}, p=0.31)$. None of the patients had advanced renal dysfunction or liver dysfunction. Median levels of aspartate aminotransferase, alanine aminotransferase, and serum creatinine were in the reference ranges of our hospital for both the EM and IM groups. The age, disease duration of schizophrenia, body weight, and the number of concomitant drugs were not significantly different between EM and IM groups. Although 15 patients (68.2\%) concomitantly used other antipsychotics except risperidone, the total of chlorpromazine equivalent dose (mg/day) was not significantly different between EM and IM groups (median: 500 (400-1010) vs. 475 (250-1800) mg, $p=0.79)$. In this study, olanzapine and levomepromazine were each used in six patients (27.3\%), aripiprazole and chlorpromazine were each used in three patients (13.6\%), quetiapine, zotepine, paliperidone, and blonanserin were each used in two patients $(9.1 \%)$, and sulpiride was used in one patient (4.5\%). None of the patients had a medical history of Parkinson's disease. Paroxetine, a potent CYP2D6 inhibitor, was concomitantly used in one patient in the EM group.

Onset of extrapyramidal symptoms (DIEPSS score $\geq 1$ ) was observed in $13(59.1 \%)$ out of the 22 patients. The DIEPSS score in the IM group was significantly higher than that in EM group (Fig. 1; median: 5.0 (3.5-6.5) vs. 0.0

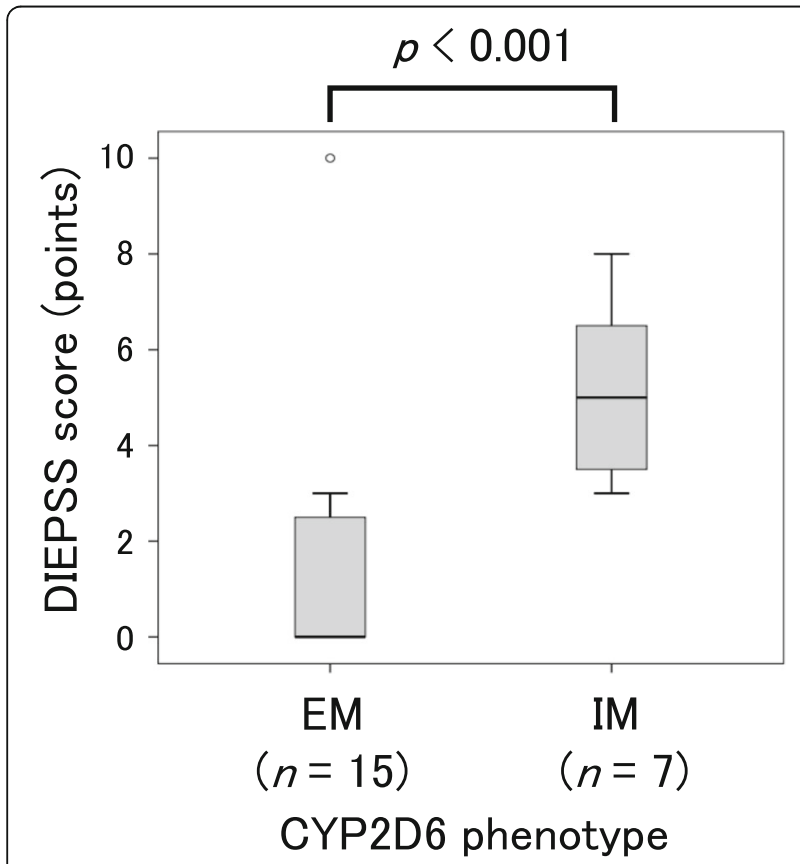

Fig. 1 Influence of CYP2D6 genotype on DIEPSS score. The box plot shows minimum, first quartile, median, third quartile, and maximum. The central rectangle spans the first quartile to the third quartile (the interquartile range). An outlier between 1.5 and 3 times the interquartile range is shown as an open circle. Statistical analysis was performed using the Mann-Whitney $U$ test
(0.0-3.0), $\quad p<0.001)$. No significant correlation was observed between the DIEPSS score and risperidone dose corrected by body weight when all patients were included in the analysis. (Fig. $2 ; \mathrm{y}=5.55 \mathrm{x}+2.31, \mathrm{R}^{2}=0.006$, $p=0.749)$. Also, no significant correlation was observed between the DIEPSS score and risperidone doses $(\mathrm{mg} / \mathrm{kg})$ when the regression analysis is conducted for each genotype (Fig. $2 ; \mathrm{y}=-34.33 \mathrm{x}+3.80, \mathrm{R}^{2}=0.176, p=0.136$ in the EM group, $y=20.85 x+3.36, R^{2}=0.297, p=0.263$ in the IM group).

Multiple regression analysis was performed to identify factors affecting the DIEPSS [DIEPSS $=3.81+0.043 \times$ age (years) $-4.15 \times$ bilirubin $(\mathrm{mg} / \mathrm{dL})-4.77 \times$ serum creatinine $(\mathrm{mg} / \mathrm{dL})+2.65 \times C Y P 2 D 6$ status $(\mathrm{IM}=1$ and $\mathrm{EM}=0)]$ (Table 2). CYP2D6 IM was proven to be a significant factor affecting the DIEPSS $(p<0.05)$.

\section{Discussion}

Among 22 patients, extrapyramidal symptoms (DIEPSS score $\geq 1$ ) were determined in 13 patients $(59.1 \%)$, demonstrating a strong association between adverse drug effects and the risperidone therapy, opposite to the relatively low frequency reported.

The DIEPSS score in the CYP2D6 IM group was significantly higher than that in EM group in this study, while we found no difference in risperidone dosing corrected by body weight between the two groups. Since adverse reactions can be correlated with risperidone concentrations, the 2017 consensus guidelines in neuropsychopharmacology recommend therapeutic drug monitoring for patients treated with risperidone [21]. To avoid neurological adverse reactions, risperidone doses which achieve over $40 \mathrm{ng} / \mathrm{mL}$ risperidone plus 9-hydroxy-risperidone plasma concentration should only be administrated in cases of insufficient or absence of therapeutic response [21]. Unfortunately, we did not

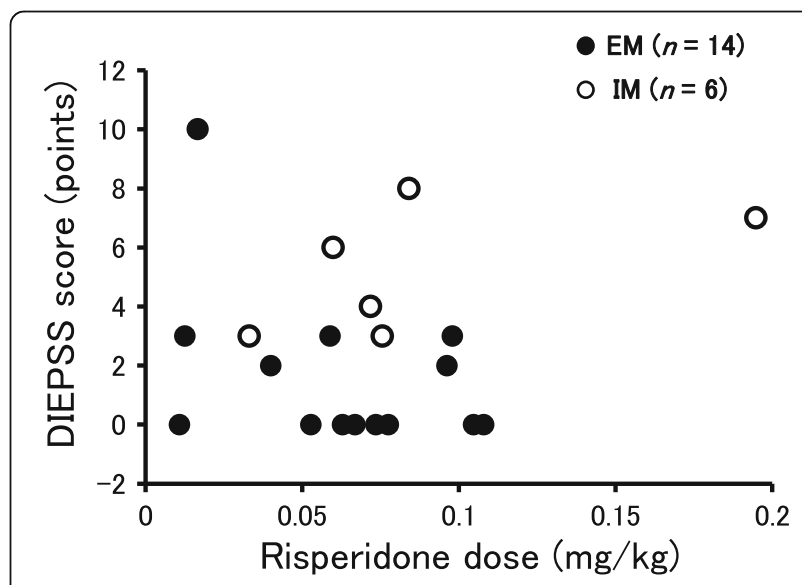

Fig. 2 Correlation between risperidone dose per body weight and DIEPSS score. Two cases were excluded because information on the body weight was not obtained 
Table 2 Predictive factors for the DIEPSS score

\begin{tabular}{llll}
\hline Factors & $\mathrm{B}$ & Std. error & $p$ \\
\hline Constant & 3.81 & 4.10 & 0.367 \\
Age & 0.043 & 0.037 & 0.265 \\
Bilirubin & -4.15 & 2.82 & 0.160 \\
Serum creatinine & -4.77 & 4.23 & 0.276 \\
CYP2D6 IM & 2.65 & 1.16 & $0.035^{*}$
\end{tabular}

The $F$ score of this model was $4.24(p=0.016)$ with adjusted $R^{2}=0.393$ $B$ unstandardized coefficients, $I M$ intermediate metabolizer

${ }^{*} p<0.05$ was considered statistically significant

perform plasma concentration measurements of risperidone and the subsequent dose adjustments in this study. On the basis of simulation results in a typical patient (body weight of $70 \mathrm{~kg}$ and creatinine clearance of 120 $\mathrm{mL} / \mathrm{min}$ ) on stable therapy with risperidone $2 \mathrm{mg}$ twice daily $(4 \mathrm{mg} /$ day), the average steady-state plasma concentration of risperidone active moiety in the IM group is approximately $40 \mathrm{ng} / \mathrm{mL}$ and 1.6-fold higher compared with patients with $C Y P 2 D 6 * 1 / * 1$ genotype [8]. Although pharmacokinetics of risperidone and its active metabolite are affected by age, gender, body weight, smoking habits, co-administered drugs, and CYP2D6 genotype [14], the patient characteristics such as age, body weight, and laboratory data were not significantly different between EM and IM groups in this study. Disease duration of schizophrenia can affect the severity of extrapyramidal symptoms, but there were no significant differences between EM and IM groups. Additionally, the results in this study were similar even if one patient who used paroxetine was excluded (data not shown). Therefore, we speculate that the decrease in the clearance of risperidone in the IM group causes an increased risperidone plasma concentration, which increases the severity of the extrapyramidal symptoms. This is the first report to show an association between DIEPSS score and CYP2D6 polymorphism.

In this study, no significant correlation was obtained between the DIEPSS score and risperidone dose corrected by the body weight when the regression analysis is conducted for all patients or each genotype. A previous study showed a weak but significant correlation between oral risperidone dose and plasma concentrations of the active moiety [22]. On the other hand, pharmacokinetics of risperidone and its active metabolite are affected by age, gender, body weight, smoking habits, and co-administered drugs in addition to CYP2D6 genotype [14]. Therefore, we consider that the sum of plasma concentrations of risperidone and 9-hydroxy-risperidone cannot be precisely predicted by the risperidone dose only. Moreover, we consider that the sample size in this study might be insufficient to detect the relationship between the DIEPSS score and risperidone doses, and there might be other risk factors of higher DIEPSS to explain why the DIEPSS score and risperidone dose are not correlated in our study.

The results of multiple regression analysis show that CYP2D6 polymorphism is the only significant factor to be correlated with the DIEPSS. Since the sample size in this study is relatively small, any effects of other previously reported factors influencing the pharmacokinetics and pharmacodynamics of risperidone remain to be clarified with a larger number of patients.

Several limitations of this study should be acknowledged. This study is a retrospective study based on medical records, and we did not measure the plasma concentration of risperidone and active metabolite 9-hydroxy-risperidone. In a future prospective study, it is necessary to clarify the relationship among CYP2D6 polymorphism, plasma concentrations of risperidone and its metabolite, pharmacological effects, and the severity of adverse effects in Japanese patients. In addition, detailed information on the duration from the start of risperidone to scoring DIEPSS was not available in this study, and 15 patients (68.2\%) concomitantly used antipsychotics beside risperidone. Therefore, a prospective study should be conducted including patients on newly risperidone monotherapy to exclude the potential influence of the duration of risperidone treatment and other antipsychotics on the result interpretation.

\section{Conclusions}

The DIEPSS score was significantly higher in the CYP2D6 IM group compared with in the EM group. The role of CYP2D6 genotyping in personalizing risperidone therapy in patients with schizophrenia should be examined in a prospective study using plasma concentration measurements of risperidone and 9-hydroxy-risperidone.

\section{Abbreviations}

ALT: Alanine aminotransferase; AST: Aspartate aminotransferase; CYP: Cytochrome P450; DIEPSS: Drug-induced extrapyramidal symptoms scale; DSM: Diagnostic and Statistical Manual of Mental Disorders; eGFR: Estimated glomerular filtration rate; EMs: Extensive metabolizers; IMs: Intermediate metabolizers; PMs: Poor metabolizers

\section{Acknowledgements}

We are grateful to all participating patients as well as cooperating physicians, pharmacists, and other medical professionals at Kobe University Hospital.

\section{Funding}

This work was supported by the Research Foundation for Pharmaceutical Sciences (Japan).

\section{Availability of data and materials}

The data will not be shared because of human data.

\section{Authors' contributions}

$\mathrm{TI}, \mathrm{KY}$, and IY conceived the study, designed the protocol. TI, KY, and FO carried out the study. TI, KY, and IY drafted the manuscript. IO and $\mathrm{AH}$ obtained the informed consent from each patient and participated in the data acquisition. $\mathrm{FO}, \mathrm{IO}, \mathrm{AH}, \mathrm{IS}$, and $\mathrm{MH}$ participated in interpretation of the data and contributed the discussions. All authors read and approved the final manuscript. 


\section{Ethics approval and consent to participate}

The present study was approved by the Ethics Committee of the Kobe University Graduate School of Medicine for Genetic Analysis (No. 57). Written informed consent was obtained from each patient registered in the study.

\section{Consent for publication}

Not applicable.

\section{Competing interests}

The authors declare that they have no competing interests.

\section{Publisher's Note}

Springer Nature remains neutral with regard to jurisdictional claims in published maps and institutional affiliations.

\section{Author details}

'Department of Pharmacy, Kobe University Hospital, 7-5-2 Kusunoki-cho, Chuo-ku, Kobe 650-0017, Japan. ${ }^{2}$ Department of Psychiatry, Kobe University Graduate School of Medicine, 7-5-1 Kusunoki-cho, Chuo-ku, Kobe 650-0017, Japan.

Received: 31 August 2018 Accepted: 7 November 2018 Published online: 19 November 2018

\section{References}

1. Delay J, Deniker P, Ropert R. Four years of experience with chlorpromazine in therapy of psychoses. Presse Med. 1956;64(22):493-6.

2. Masi G, Liboni F. Management of schizophrenia in children and adolescents: focus on pharmacotherapy. Drugs. 2011;71(2):179-208.

3. Caroff SN, Hurford I, Lybrand J, Campbell EC. Movement disorders induced by antipsychotic drugs: implications of the CATIE schizophrenia trial. Neurol Clin. 2011;29(1):127-48.

4. Kose $E$, Uno $K$, Hayashi $H$. Evaluation of the expression profile of extrapyramidal symptoms due to antipsychotics by data mining of Japanese adverse drug event report (JADER) database. Yakugaku Zasshi. 2017;137(1):111-20.

5. de Araújo AA, de Araújo Dantas D, do Nascimento GG, Ribeiro SB, Chaves KM, de Lima Silva $V$, et al. Quality of life in patients with schizophrenia: the impact of socio-economic factors and adverse effects of atypical antipsychotics drugs. Psychiatr Q. 2014;85(3):357-67.

6. Uçok A, Gaebel W. Side effects of atypical antipsychotics: a brief overview. World Psychiatry. 2008;7(1):58-62.

7. Arranz MJ, de Leon J. Pharmacogenetics and pharmacogenomics of schizophrenia: a review of last decade of research. Mol Psychiatry. 2007; 12(8):707-47.

8. Locatelli I, Kastelic M, Koprivsek J, Kores-Plesnicar B, Mrhar A, Dolzan V, et al. A population pharmacokinetic evaluation of the influence of CYP2D6 genotype on risperidone metabolism in patients with acute episode of schizophrenia. Eur J Pharm Sci. 2010;41(2):289-98.

9. Cabaleiro T, Ochoa D, Román M, Moreno I, López-Rodríguez R, Novalbos J, et al. Polymorphisms in CYP2D6 have a greater effect on variability of risperidone pharmacokinetics than gender. Basic Clin Pharmacol Toxicol. 2015;116(2):124-8.

10. Ebisawa A, Hiratsuka M, Sakuyama K, Konno Y, Sasaki T, Mizugaki M. Two novel single nucleotide polymorphisms (SNPs) of the CYP2D6 gene in Japanese individuals. Drug Metab Pharmacokinet. 2005;20(4):294-9.

11. Kubota T, Yamaura Y, Ohkawa N, Hara H, Chiba K. Frequencies of CYP2D6 mutant alleles in a normal Japanese population and metabolic activity of dextromethorphan O-demethylation in different CYP2D6 genotypes. Br J Clin Pharmacol. 2000;50(1):31-4.

12. Yoo HD, Cho HY, Lee SN, Yoon H, Lee YB. Population pharmacokinetic analysis of risperidone and 9-hydroxyrisperidone with genetic polymorphisms of CYP2D6 and ABCB1. J Pharmacokinet Pharmacodyn. 2012;39(4):329-41.

13. Spina $E$, de Leon J. Clinical applications of CYP genotyping in psychiatry. J Neural Transm (Vienna). 2015;122(1):5-28.

14. Urban AE, Cubała WJ. Therapeutic drug monitoring of atypical antipsychotics. Psychiatr Pol. 2017;51(6):1059-77.

15. Inada T. Evaluation and diagnosis of drug-induced extrapyramidal symptoms: commentary on the DIEPSS and guide to its usage. Tokyo: Seiwa Shoten Publishers; 1996.
16. Inada T, Yagi G, Miura S. Extrapyramidal symptom profiles in Japanese patients with schizophrenia treated with olanzapine or haloperidol. Schizophr Res. 2002;57(2-3):227-38.

17. Inada T. A second-generation rating scale for antipsychotic-induced extrapyramidal symptoms: drug-induced extrapyramidal symptoms scale. Tokyo: Seiwa Shoten Publishers; 2009.

18. Kim JH, Jung HY, Kang UG, Jeong SH, Ahn YM, Byun HJ, et al. Metric characteristics of the drug-induced extrapyramidal symptoms scale (DIEPSS): a practical combined rating scale for drug-induced movement disorders. Mov Disord. 2002;17(6):1354-9.

19. Association AP. Diagnostic and Statistical manual of mental disorders. fourth ed. Washington, DC: American Psychiatric Association; 1994.

20. Okubo M, Murayama N, Miura J, Shimizu M, Yamazaki H. A rapid multiplex PCR assay that can reliably discriminate the cytochrome P450 2D6 whole-gene deletion allele from 2D6*10 alleles. Clin Chim Acta. 2012:413(19-20):1675-7.

21. Hiemke C, Bergemann N, Clement HW, Conca A, Deckert J, Domschke K, et al. Consensus guidelines for therapeutic drug monitoring in Neuropsychopharmacology: update 2017. Pharmacopsychiatry. 2018;51(102):9-62.

22. Riedel M, Schwarz MJ, Strassnig M, Spellmann I, Müller-Arends A, Weber K, et al. Risperidone plasma levels, clinical response and side-effects. Eur Arch Psychiatry Clin Neurosci. 2005;255(4):261-8.

\section{Ready to submit your research? Choose BMC and benefit from:}

- fast, convenient online submission

- thorough peer review by experienced researchers in your field

- rapid publication on acceptance

- support for research data, including large and complex data types

- gold Open Access which fosters wider collaboration and increased citations

- maximum visibility for your research: over $100 \mathrm{M}$ website views per year

At BMC, research is always in progress.

Learn more biomedcentral.com/submissions 